Pacific Journal of Mathematics

DERIVABLE SEMI-TRANSLATION PLANES 


\title{
DERIVABLE SEMI-TRANSLATION PLANES
}

\author{
NORMAN L. JOHNSON
}

\begin{abstract}
The author has previously classified semi-translation planes with respect to $\left(p, l, \pi_{0}\right)$-transitivity. This paper is concerned with the determination of the types of semi-translation planes that can be constructed from certain dual translation planes by Ostrom's method of "derivation".
\end{abstract}

If $\pi$ is a derivable plane that is $\left((\infty), L_{\infty}\right)$-transitive then the plane derived from $\pi$ is a semi-translation plane. There are only two known classes of planes that are $\left((\infty), L_{\infty}\right)$-transitive but not $(p, L)$ transitive for any additional pointline pair. They are the OstromRosati planes (and their duals) and the planes derived from the dual Lüneburg planes. With the exception of the above planes and the translation planes, the only known derivable planes that are $\left((\infty), L_{\infty}\right)$ transitive are dual translation planes. Ostrom has shown that if $\pi$ is a strict semi-translation plane of order $q^{2}$ and $q>4$, which is derived from a dual translation plane whose coordinate system is of dimension 2 over its (left) kernel, then the full collineation group of the projective extension of $\pi$ fixes the line at infinity of $\pi$. We shall give a slightly different proof of Ostrom's theorem which also includes the case $q=4$.

The author has previously classified semi-translation planes with respect to $\left(p, L, \pi_{0}\right)$-transitivity. With the exception of the Hughes planes, for every known example of a semi-translation plane belonging to a certain class there is an example of a semi-translation plane in the same class and which is derived from a dual translation plane. This suggests that one might look, in general, at the possible semitranslation planes so derived.

Ostrom has shown that the number of possible translation planes (and hence dual translation planes) is extremely large. This suggests that the number of distinct semi-translation planes derived from dual translation planes may also be very large. However, relative to our classification, we will show that the number of classes of such planes is exactly five.

2. Background material. We shall present the essential material necessary for reading this article. However, the reader is referred to Ostrom's papers [10], [15], [17], [18] and [19] for a complete background.

Definition 2.1. Let $V$ be a vector space over a field $F$. Let 
$N(V)$ be the following system of points and lines: the points of $N(V)$ are the ordered pairs $(x, y)$ of elements of $V$. For each $c \in V$, the set of points $(x, y)$ such that $x=c$, is a line. For each $\alpha \in F$ and for each $b \in V$, the set of points $(x, y)$ such that $y=x \alpha+b$, is a line (see [18], p. 5). In this case, $N(V)$ will be said to be a derivable net.

Note that we may coordinatize a net by Hall's method (see [18]) with the restriction that since we may not have enough parallel classes to form an affine plane, multiplication may not be defined for all elements of the coordinate system.

If $F$ is $G F(q)$ and $V$ is of dimension $r$ over $F$, then $N(V)$ is of order $q^{r}$. Also, the number of parallel classes of $N(V)$ is $q+1$.

Definition 2.2. A net $N$ is said to be replaceable if there is a net $N^{\prime}$ defined on the same points such that each pair of points that is collinear in $N$ is also collinear in $N^{\prime}$, and vice-versa. We shall call $N^{\prime}$ a replacement for $N$.

The derivable net $N(V)$ of (2.1) is a replaceable net if and only if $V$ is of dimension 2 over $F$ (see [18]).

Let $N(V)$ be embedded in an affine Desarguesian plane $\pi$ of order $q^{2}$. That is, $N(V)$ and $\pi$ are defined on the same points and each line of $N(V)$ is a line of $\pi$. Let $(C,+, \cdot)$ be the ternary ring of $\pi$. Hence, $(C,+)$ is a vector space of dimension 2 over $F$ relative to the product $x \alpha$ as a scalar product. Let $t \in C$ and $\notin F$, then every element of $C$ may be uniquely represented in the form $t \alpha+\beta$ for $\alpha, \beta \in F$ (we identify the vector $1 \cdot \alpha$ with $\alpha \in F$ ).

Let $\pi_{0}$ be the subplane of $\pi$ coordinatized by $F=G F(q)$. That is, points of $\pi_{0}$ are of the form $(\alpha, \beta)$ for $\alpha, \beta \in F$. Consider the images of $\pi_{0}$ under the mappings $(x, y) \rightarrow(a x, a y)$ for all nonzero $a \in C$. The translates of these images are the lines of a net $N^{\prime}(V)$ that is a replacement for $N(V)$ (see [18]).

Definition 2.3. Let $N(V) \cup M, M$ a net defined on the same points as $N(V)$, indicate the incidence structure of the common points of $N(V)$ and $M$ and the lines of $N(V)$ and of $M$. Also, $N(V)$ and $M$ are assumed to be disjoint; i.e., two points joined by a line of $N(V)$ are not joined by a line of $M$ and conversely. By (3.3) [18], $N(V) \cup M$ is a net. An affine plane $\pi$ is said to be derivable if it is of the form $N(V) \cup M$ with $V$ of dimension 2 over $F$. If $\pi^{\prime}=N^{\prime}(V) \cup M$ (see above remarks) then $\pi^{\prime}$ will be said to be derived from $\pi$. (Ostrom [15] has actually shown that weaker conditions than the above are 
sufficient for derivation, but we know of no planes admitting these weaker (but not the stronger) conditions.)

Derivation is an involutory process. This is suggested by the fact that lines of $N^{\prime}(V)$ are subplanes of $N(V)$ and vice-versa.

DEFINITION 2.4. Let $S$ be a coordinate system (see [18]) with associative and commutative addition. If $S$ contains a subfield $F$ such that (1) $a(\alpha+\beta)=a \alpha+a \beta,(2)(\alpha \alpha) \beta=a(\alpha \beta)$, and (3) $(a+b) \alpha=a \alpha+b \alpha$ for all $a, b \in S$ and for all $\alpha, \beta \in F$, we shall say that $S$ is a right vector space over $F$.

If $T$ is the ternary function of $S$ and if $T(x, \alpha, b)=x \alpha+b$ for all $b \in S$ and for all $\alpha \in F$, we shall say that $S$ is linear with respect to $F$.

Now let $S$ be a coordinate system which is a right vector space over a subfield $F$. Let $S$ be linear with respect to $F$ and let the dimension of $S$ over $F$ be 2 . If the coordinate system of a plane $\pi$ is as above, then the plane $\pi$ is derivable (see (2.1) and [10]).

If $Q$ is a right (left) quasifield, it is well known that $Q$ may be used to coordinatize a (dual) translation plane. The kernel $F$ of $Q$ is a skewfield and if $Q$ is a left quasifield then $Q$ is a right vector space over $F$. If $\pi$ is a dual translation plane with respect to $(\neg)$ then the ternary function is linear (see [17]). Therefore, if the coordinate system of a dual translation plane of order $q^{2}$ (with respect to $(x)$ ) is of dimension 2 over its (left) kernel then $\pi$ is derivable (that is, the affine version of $\pi$ is derivable). (We are using $L_{\infty}$ to denote the line at infinity and $(\infty)=L_{\infty} \cap\{(x, y) \mid x=$ constant $\}$.)

THEOREM 2.5. (Ostrom [15]). We may choose a coordinate system $C$ for a semi-translation plane $\pi$ (of order $q^{2}$ ) which contains a subsystem $F$ which is a quasifield of order $q$ such that

(1) points of $\pi_{0}$ have coordinates in $F$ for some subplane $\pi_{0}$ of $\pi$ of order $q$,

(2) lines of $\pi_{0}$ have equations of the type $y=x \alpha+\beta$ or $x=\beta$ for all $\alpha, \beta \in F$,

(3) lines of $\pi$ whose slopes $m$ are not in $F$ have equations of the form : $y=(x-\beta) m+\alpha$ for $\alpha, \beta \in F$,

(4) $(x-\alpha) \beta=x \beta-\alpha \beta$ for all $x \in C$ and for all $\alpha, \beta \in F$,

(5) if $c \notin F$ and $d \in C$ there exist unique $\alpha, \beta \in F$ such that $d=$ $c \alpha+\beta$.

Theorem 2.6. (Albert [1]). Let $\pi$ be a derivable plane (recall $\pi$ is 
now considered as an affine plane). Let $t$ be an element of the coordinate system $C$ of $\pi$ such that $t \notin F$ (see (2.1), (2.2), (2.4)). Then the derived plane $\pi^{\prime}$ can be coordinatized by a coordinate system $C^{\prime}$ such that a point with coordinates $(x, y)=\left(t x_{1}+x_{2}, t y_{1}+y_{2}\right)$ in $C$ has coordinates $\left(x^{\prime}, y^{\prime}\right)=\left(t x_{1}+y_{1}, t x_{2}+y_{2}\right)$ in $C^{\prime}$.

Note that $\pi_{0}=\{(\alpha, \beta) ; \alpha, \beta \in F\}$ in $\pi$ has coordinates $\{(0, t \alpha+\beta)$; $\alpha, \beta \in F\}$ in $C^{\prime}$. That is, the set of points of the subplane $\pi_{0}$ in $\pi$ is the same as the set of points of $x^{\prime}=0$ in $\pi^{\prime}$.

A collineation of $\pi$ which fixes $N(V)$ also fixes $M$ (see (2.3)). Such a collineation induces a collineation of $\pi^{\prime}$. In particular, a translation of $\pi$ induces a translation of $\pi^{\prime}$ (Theorem 7 [15], (4.8) [18]).

Let $L$ be a line of $\pi$ which is not a line of $\pi^{\prime}$. If $\pi$ admits a group of translations transitive on the points of $L$, then the projective extension of $\pi^{\prime}$ is a semi-translation plane with respect to $L_{\infty}^{\prime}$. Conversely, if $\pi^{\prime}$ is a semi-translation plane there exists such a line $L$ in $\pi$ (corollary to Theorem 7 [15]).

Let $\underline{\pi}$ be a dual translation plane (order $q^{2}$ ) with respect to $(\infty)$ and whose coordinate system is a right 2-dimensional vector space over $G F(q)$. Thus, $\underline{\pi}$ is derivable and if $x=0$ in $\pi$ is the same as the set of points of $\pi_{0}^{\prime}$ ( $\pi_{0}^{\prime}$ some subplane of the derived plane of $\pi^{\prime}$ ) then by the previous remarks, $\underline{\pi}^{\prime}$ is a semi-translation plane. As pointed out in the introduction, it will be shown that the full collineation group of the projective extension of $\underline{\pi}^{\prime}$ fixes $L_{\infty}^{\prime}$.

Note that if $\left(t x_{1}+x_{2}, t y_{1}+y_{2}\right) \rightarrow\left(t z_{1}+z_{2}, t w_{1}+w_{2}\right)$ is a collineation of the derivable semi-translation plane $\pi$ which fixes $M, x_{i}, y_{i}, z_{i}, w_{i} \in F$, $i=1,2$, then, by (2.6), $\left(t x_{1}+y_{1}, t x_{2}+y_{2}\right) \rightarrow\left(t z_{1}+w_{1}, t z_{2}+w_{2}\right)$ is a collineation of $\pi^{\prime}$ ( $\pi^{\prime}$ the plane derived from $\pi$ ).

THEOREM 2.7. (Ostrom [15]). Let $\pi$ be a derivable semi-translation plane coordinatized as in (2.6), then:

(a) Addition in $C$ is isomorphic to addition in $C^{\prime}$.

(b) $F^{\prime}$ is a subsystem of $C$.

(c) $C$ is linear with respect to $F^{\prime}$.

(d) Multiplication on the right by elements of $F^{\prime}$ is the same in $C$ and $C^{\prime}$.

(e) If $\cdot$ denotes multiplication in $C^{\prime}$ and $\circ$ denotes multiplication in $C$, then: $\left(t x_{1}+y_{1}\right) \circ\left(t z_{1}+z_{2}\right)=t x_{2}+y_{2}$ if and only if $\left(t x_{1}+x_{2}\right)$. $\left(t u_{1}+u_{2}\right)=t y_{1}+y_{2}$ where $z_{1} \cdot\left(t u_{1}+u_{2}\right)=t+z_{2}$, provided $z_{1} \neq 0$ and $x_{i}, y_{i}, z_{i}, u_{i} \in F, i=1,2$.

DEFINITION 2.8. We shall say that a system $Q$ satisfying the following properties is automorphic: Let $Q$ be a set of $q^{2}$ elements with the operations of + and $\cdot$ such that: 
(1) Addition is an Abelian group.

(2) Nonzero elements of $Q$ form a loop under multiplication.

(3) $Q$ contains a subsystem $F$ which is a field of order $q$ with respect to addition and multiplication.

(4) $Q$ is a right vector space of dimension 2 over $F$ with respect to multiplication on the right by elements of $F$.

(5) $a(b+\alpha)=a b+a \alpha$ for all $a, b \in Q$ and for all $\alpha \in F$.

(6) $Q$ admits a group of automorphisms of order $q$ which fixes each element of $F$. The group maps $t \rightarrow t+\beta$ for all $\beta \in F$ where $t$ and 1 are basis elements for the vector space.

Notice that the coordinate system $S$ for a dual translation plane (of order $q^{2}$ whose kernel is $G F(q)$ ) with respect to $(\infty)$ is automorphic if $S$ admits automorphisms of the form $t \rightarrow t+\beta$ for each $\beta \in F$ which fix the kernel elementwise.

THEOREM 2.9. (Morgan and Ostrom [8]). If $C$ is an automorphic system and if $\left(t \alpha_{1}\right)\left(t \alpha_{2}\right)=t h\left(\alpha_{1}, \alpha_{2}\right)+k\left(\alpha_{1}, \alpha_{2}\right)$ where $\alpha_{1} \neq 0$ and $h\left(\alpha_{1}, \alpha_{2}\right), k\left(\alpha_{1}, \alpha_{2}\right) \in F$, then: $\left(t \alpha_{1}+\beta_{1}\right)\left(t \alpha_{2}+\beta_{2}\right)=t\left[h\left(\alpha_{1}, \alpha_{2}\right)-\alpha_{2} \beta_{1}+\alpha_{1} \beta_{2}\right]$ $+\beta_{1} \alpha_{1}^{-1} h\left(\alpha_{1}, \alpha_{2}\right)+k\left(\alpha_{1}, \alpha_{2}\right)-\beta_{1}^{2} \alpha_{1}^{-1} \alpha_{2}+\beta_{1} \beta_{2}$.

THEOREM 2.10. (Ostrom [15]). Let $\pi$ be a derivable plane and let the set of points such that $x=0$ in $\pi$ be the same as the set of points of the subplane $\pi_{0}^{\prime}$ in $\pi^{\prime}$ ( $\pi^{\prime}$ indicating the plane derived from $\pi$ ). If $\beta$ is a collineation of $\pi$ which fixes the set of points $(x, y)$ such that $x=0$ pointwise and fixes $M$ (see (2.3)) then $\beta$ induces an automorphism of $C^{\prime}$ which fixes $F^{\prime}$ pointwise.

AsSumption 2.11. We will assume for the remainder of this article that $\pi$ is a strict semi-translation plane which is derived from a dual translation plane $\underline{\pi}$ with respect to $(\infty)$ whose coordinate system is of dimension 2 over its kernel and which is coordinatized as in (2.6) and (2.7). We denote the coordinate systems of $\pi$ and $\underline{\pi}$ by $(C,+, \cdot)$ and $(C,+, *)$, respectively. The subfield $\underline{F}$ of $C$ is actually the same as the subfield $F$ of $C$ and so we will not distinguish between $F$ and $\underline{F}$ (see (2.7)).

Notice that if we have a semi-translation plane with respect to a line $L$ (see [4]), we can choose $L$ to be the line that is deleted in obtaining an affine plane. That is, we may choose $L=L_{\infty}$. Also, we may choose $\pi_{0}$ as the subplane coordinatized by the subfield $F$. This amounts to choosing a line of $\underline{\pi}$ and calling it $x=0$. Also, the choice of $t$ in $\pi$ depends on the arbitrary choice of $(0)$ in $\pi$. That is, $y=0$ in $\pi$ is the set of points $(x, y)$ such that $x=t \alpha, y=t \beta$ in $\underline{\pi}$. (Note 
that we are denoting the lines $\{(x, y) \mid y=f(x)\}$ by $y=f(x)$.)

Definition. 2.12. Let $\Sigma^{*}$ be a projective plane of order $q^{2}$ and $\Sigma_{0}^{*}$ a projective subplane of order $q$. Let $p$ be a point of $\Sigma_{0}^{*}$ and $L$ a line of $\Sigma^{*}$ such that $L \cap \Sigma_{0}^{*}$ is a line of $\Sigma_{0}^{*}$. $\Sigma^{*}$ is said to be $\left(p, L, \Sigma_{0}^{*}\right)$-transitive if the stabilizer of $\Sigma_{0}^{*}$ in the group of all $(p, L)$ collineations of $\Sigma^{*}$ induces a collineation group of $\Sigma_{0}^{*}$ such that $\Sigma_{0}^{*}$ is $(p, L)$-transitive. Let $\Sigma$ be an affine plane of order $q^{2}$ and $\Sigma_{0}$ an affine subplane of order $q$ of $\Sigma$. Let $\Sigma^{*}$ and $\Sigma_{0}^{*}$ be the projective extensions of $\Sigma$ and $\Sigma_{0}$, respectively. We shall say that $\Sigma$ is $\left(p, L, \Sigma_{0}\right)$-transitive if and only if $\Sigma^{*}$ is $\left(p, L, \Sigma_{0}^{*}\right)$-transitive.

Thus, a semi-translation plane $\pi$ is $\left(p, L_{\infty}, \pi_{0}\right)$-transitive for all points $p \in L_{\infty} \cap \pi_{0}^{*}$ for some subplane $\pi_{0}^{*}$ of $\pi^{*}\left(\pi^{*}, \pi_{0}^{*}\right.$ the projective extensions of $\pi, \pi_{0}$, respectively). Hereafter we do not formally distinguish between $\pi_{0}$ ane $\pi_{0}^{*}$.

The following mappings represent collineations of the dual translation plane $\pi$ :

(i ) $(x, y) \rightarrow(x, y+a)$ for all $a \in \underline{C}$. These are the $q^{2}$ translations with center $(\alpha)$.

(ii) $(x, y) \rightarrow(x, y \alpha)$ for all nonzero $\alpha \in \underline{F}$; $\left((\times), y=0, \pi_{0}\right)$-transitivity (see (3.4)). These mappings are collineations since $\underline{C}$ is of dimension 2 over $\underline{F}$ (see proof of (3.6)).

(iii) $(x, y) \rightarrow(x, x a+y)$ for all $a \in \underline{C} ;((\alpha), x=0)$-transitivity (see proof of (3.2)).

The mappings (iii) for $a \in F$ induce an automorphism group of order $q$ fixing $F$ pointwise in $C$ (see (2.10)). It is an easy exercise to see that these automorphisms are represented by $t \rightarrow t+\alpha$ for all $\alpha \in F$ (see (2.6) and the remarks immediately following).

Note also that the relations (2.6) and (2.7) between the coordinate systems for $\pi$ and $\pi$ are reciprocal since the deriving process is involutory.

3. The possible types of semi-translation planes derived from dual translation planes. The following five lemmas are very useful and so are included here. Their proofs are very similar to the corresponding theorems for $(p, L)$-transitivity, so we will merely sketch the proofs.

Lemma 3.1. If $\pi$ is $\left((0), x=0, \pi_{0}\right)$-transitive then $C$ is such that $c(\alpha m)=(c \alpha) m$ for all $c, m \in C$ and for all $\alpha \in F$. Also, $(x, y) \rightarrow(x \alpha, y)$ for all nonzero $\alpha \in F$ represents these collineations.

Proof. For each $\alpha \in F \exists\left((0), x=0, \pi_{0}\right)$-collineation fixing $\pi_{0}$ such that $(1, m) \rightarrow(\alpha, m)$ and $(0,0) \rightarrow(0,0)$. Therefore, $y=x m \rightarrow y=$ 
$x \underline{m} \ni \alpha \underline{m}=m$. If we let $x=c \rightarrow x=c^{\prime}$ it follows that $(c, c m) \rightarrow\left(c^{\prime}, c m\right)$ so that $c^{\prime} \underline{m}=c m$. But $m=\alpha \underline{m}$, which implies $c^{\prime} m=c(\alpha \underline{m})$. Letting $m=\alpha$ implies $\underline{m}=1$ and $c^{\prime}=c \alpha$. Thus, $(c \alpha) \underline{m}=c(\alpha \underline{m})$ for all $c, \underline{m} \in C$ and for all $\alpha \in F$.

Lemma 3.2. If $\pi$ is $\left((\infty), x=0, \pi_{0}\right)$-transitive then $C$ is such that $c(\alpha+m)=c \alpha+c m$ for all $c, m \in C$ and for all $\alpha \in F$. Also, these collineations are represented by $(x, y) \rightarrow(x, x \beta+y)$ for all $\beta \in F$.

Proof. For each $\beta \in F^{\exists}\left((\infty), x=0, \pi_{0}\right)$-collineation carrying (0) to $(\beta)$. It follows that this collineation may be represented by the mapping $(x, y) \rightarrow(x, x \beta+y)$. The assertion of (3.2) is then clear.

Lemma 3.3. If $\pi$ is $\left((0,0), L_{\infty}, \pi_{0}\right)$-transitive then $C$ has the following properties: (1) $(\alpha c) m=\alpha(\mathrm{cm})$ for all $c, m \in C$ and for all $\alpha \in F$. (2) $\alpha(c+m)=\alpha c+\alpha m$ for all $c, m \in C$ and for all $\alpha \in F$. Also, $(x, y) \rightarrow(\alpha x, \alpha y)$ for all nonzero $\alpha \in F$ represents these collineations.

Proof. For each $\alpha \in F-\{0\}$ there is a $\left((0,0), L_{\infty}, \pi_{0}\right)$-collineation such that $(1,1) \rightarrow(\alpha, \alpha)$. Using the fact that points on $L_{\infty}$ and lines through $(0,0)$ are fixed, we can establish that $(1, m) \rightarrow(\alpha, \alpha m)$ and $y=m \rightarrow y=\alpha m$.

Thus, $(c, c m) \rightarrow(\bar{c}, \alpha(\mathrm{cm}))$ such that $\bar{c} m=\alpha(\mathrm{cm})$ for all $c, m \in C$. It then follows that the collineations are represented by the mappings $(x, y) \rightarrow(\alpha x, \alpha y)$ from which the result immediately follows.

Lemma 3.4. If $\pi$ is $\left((\infty), y=0, \pi_{0}\right)$-transitive then $(\mathrm{cm}) \alpha=c(m \alpha)$ for all $c, m \in C$ and for all $\alpha \in F$. Also, $(x, y) \rightarrow(x, y \alpha)$ for all $\alpha \in F$ represents these collineations.

\section{Proof. Lemma 4 [9].}

Lemma 3.5. If $\pi$ is $\left((\infty), y=0, \pi_{0}\right)$-and $\left((0), x=0, \pi_{0}\right)$-transitive and $\alpha a=a \alpha$ for all $\alpha \in F$ and for all $\alpha \in C$, then $\pi$ is also $\left((0,0), L_{\infty}, \pi_{0}\right)$-transitive.

Proof. From (3.4) and (3.1) we have collineations represented by $(x, y) \rightarrow(x \alpha, y)$ and $(x, y) \rightarrow(x, y \alpha)$. Thus we have collineations represented by $(x, y) \rightarrow(\alpha x, \alpha y) \forall \alpha \in F-\{0\}$. (3.5) then follows from (3.4).

LeMMA 3.6. Let $\underline{\pi}$ be recoordinatized in either of the following two ways: 
(i) a point with coordinates $\left(t x_{1}+y_{1}, t x_{2}+y_{2}\right)$ will have coordinates $\left(t y_{1}+x_{1}, t y_{2}+x_{2}\right)$;

(ii) a point with coordinates $\left(t x_{1}+y_{1}, t x_{2}+y_{2}\right)$ will have coordinates $\left(t\left(x_{1}+y_{1} \alpha\right)+y_{1}, t\left(x_{2}+y_{2} \alpha\right)+y_{2}\right)$ for some $\alpha \in F ; x_{i}, y_{i} \in F$, $i=1,2$. Then $\pi$ remains a dual translation plane with respect to ( ) whose coordinate system is of dimension 2 over its kernel.

Proof. ( i ) Let $\left(x^{\prime}, y^{\prime}\right)$ denote coordinates in the new coordinate system.

Clearly, the sets of points satisfying the equations $x^{\prime}=c, y^{\prime}=c^{\prime \prime}$, and $y^{\prime}=x^{\prime}$ are lines so that we have a legitimate coordinate system ((2.4) [18]).

A plane $\pi$ is a dual translation plane (of order $q^{2}$ ) with respect to $(\infty)$ and its coordinate system is of dimension 2 over its kernel if and only if $\pi$ is $((\infty), L)$-transitive for all lines $L I(\infty)$ and $\pi$ admits collineations of the form $\left(x^{\prime}, y^{\prime}\right) \rightarrow\left(x^{\prime}, y^{\prime} \alpha\right)$ for all $\alpha \in F=G F(q)$. That is, the coordinate system $(\underline{C}+, \cdot)$ of a dual translation plane is always a vector space over its kernel. Furthermore, the kernel- $\{0\}$ is isomorphic to the group of collineations of the form $\left(x^{\prime}, y^{\prime}\right) \rightarrow\left(x^{\prime}, y^{\prime} \alpha\right)$ if $\pi$ is a dual translation plane with respect to $(\infty)$. (In this case, $y^{\prime}=x^{\prime} m+b \rightarrow y^{\prime}=x^{\prime}(m \alpha)+b \alpha$ under the above collineations so that $(c, c m+b) \rightarrow(c(c m+b) \alpha)$ which implies $c(m \alpha)+b \alpha=(c m+b) \alpha$ for all $\alpha \in F-\{0\}$ and $c, m, b \in C$.) Thus, if $\pi$ admits collineations of the form $\left(x^{\prime}, y^{\prime}\right) \rightarrow\left(x^{\prime}, y^{\prime} \alpha\right)$ for all $\alpha \in F-\{0\}$ then $\underline{C}$ is a vector space over $F$ and hence is of dimension 2 over $F$. Let the coordinate change (i) be denoted by $\sigma$.

Let $(x, y)$ denote coordinates of $\underline{C},\left(x^{\prime}, y^{\prime}\right)$ coordinates of $\underline{C} \sigma$. Let $(\underline{C},+, \cdot) \sigma=(\underline{C} \sigma, \oplus, \odot)$. Thus, $(x, y) \sigma=\left(t x_{1}+y_{1}, t x_{2}+y_{2}\right) \sigma=$ $\left(t y_{1}+x_{1}, t y_{2}+x_{2}\right)=\left(x^{\prime}, y^{\prime}\right) . \quad\{(x, y) \mid x=0\} \sigma=\left\{\left(t x_{1}+y_{1}, t x_{2}+y_{2}\right) \mid x_{1}=y_{1}=\right.$ $0\} \sigma=\left\{\left(t y_{1}+x_{1}, t y_{2}+x_{2} \mid y_{1}=x_{1}=0\right\}=\left\{\left(x^{\prime}, y^{\prime}\right) \mid x^{\prime}=0\right\}\right.$ and the line $x=c$ is $x^{\prime}=c^{\prime}$ under $\sigma$ so $(\propto)$ is fixed under the coordinate change and the set of $((\infty), L)$-transitivities $L I(\infty)$ is carried into itself.

Now $(y=0) \sigma=\left(y^{\prime}=0\right)$ so $(0) \sigma=(0)$. Thus, $0 \oplus a=a \forall a \in \underline{C} \sigma$. Also, clearly, $(1) \sigma=(1) . \quad(t \beta, t \beta \oplus \alpha) \in y^{\prime}=x^{\prime} \oplus \alpha$. Let $t \beta \oplus \alpha=t s_{1}+s_{2}$ for some $s_{1}, s_{2} \in F$. Then $y^{\prime}=x^{\prime} \oplus \alpha=(0, \alpha) \cup(1)$ if and only if $\left(\beta, t s_{2}+s_{1}\right) \in(0, t \alpha) \cup(1)$ in $(x, y)$-coordinates. That is, $\left(\beta, t s_{2}+s_{1}\right) \in y$ $=x+t \alpha$. Hence, $\beta+t \alpha=t s_{2}+s_{1} \Rightarrow s_{2}=\alpha$ and $s_{1}=\beta$. Therefore, $t \beta \oplus \alpha=t \beta+\alpha$.

Also, $(a, a \alpha) \sigma=\left(t \alpha_{1}+\alpha_{2},\left(t \alpha_{1}+\alpha_{2}\right) \alpha\right) \sigma$, for some $\alpha_{1}, \alpha_{2} \in F$, $\left(t \alpha_{1}+\alpha_{2}, t\left(\alpha_{1} \alpha\right)+\alpha_{2} \alpha\right) \sigma=\left(t \alpha_{2}+\alpha_{1}, t\left(\alpha_{2} \alpha\right)+\alpha_{1} \alpha\right)$. Also, $(0,0) \sigma=(0,0)$, $(1, \alpha) \sigma=(t, t \alpha)$ and $(t, t \alpha) \sigma=(1, \alpha)$ so that $(y=x \alpha) \sigma=\left(y^{\prime}=x^{\prime} \odot \alpha\right)$.

Hence, $\left(t \alpha_{2}+\alpha_{1}\right) \odot \alpha=\left(t \alpha_{2}+\alpha_{1}\right) \alpha$ and so $t \odot \beta_{1} \oplus \beta_{2}=t \beta_{1}+\beta_{2}$ $\forall \beta_{1}, \beta_{2} \in F$. 
Now let $\rho$ denote a collineation of $\underline{\pi}(x, y) \rightarrow(x, y \alpha)$. Letting $x=t x_{1}+x_{2}$ and $y=t y_{1}+y_{2}$, for some $x_{1}, x_{2}, y_{1}, y_{2} \in F,\left(t x_{1}+x_{2}, t y_{1}+y_{2}\right) \sigma$ $\stackrel{\rho}{\rightarrow}\left(t x_{1}+x_{2}, t\left(y_{1} \alpha\right)+\left(y_{2} \alpha\right)\right) \sigma$. So, $\left.\left(t x_{2}+x_{1}, t y_{2}+y_{1}\right) \stackrel{\rho}{\rightarrow}\left(t x_{2}+x_{1}, t\left(y_{2} \alpha\right)+y_{1} \alpha\right)\right)$ and thus $\left(x^{\prime}, y^{\prime}\right) \rightarrow\left(x^{\prime}, y^{\prime} \odot \alpha\right)$. Hence ( $\left.\mathrm{i}\right)$ is proved.

The proof of (ii) is very similar to proof of (i) and is left for the reader.

COROLLARY 3.7. The plane $\pi$ remains a derivable semi-translation plane derived from a dual translation plane $\pi$ whose coordinate system is of dimension 2 over its kernel under the coordinate change (i ) $(x, y) \rightarrow(y, x)$ or (ii) $(x, y) \rightarrow(x+y \alpha, y)$ for some $\alpha \in F$.

\section{Proof. Immediate from (3.6) and (2.6).}

Lemma 3.8. Let $\pi$ be (p, $\left.\underline{\boldsymbol{L}}, \pi_{0}\right)$-transitive with $p \in L_{\infty}$. Then we can choose $p$ and $\underline{L}$ so that $\pi$ is (i ) $\left((\infty), y=0, \pi_{0}\right)$-or $\left((0), x=0, \pi_{0}\right)$ transitive if $p \notin \underline{L}$; (ii) $\left((\infty), x=0, \pi_{0}\right)$-or $\left((0), y=0, \pi_{0}\right)$-transitive if $\underline{p} \in \underline{L}$.

Proof. (i) Suppose $p=(\infty)$ and $\underline{L}=(y=0)$. Then, by (3.7) (i), we may obtain $\left((0), x=0, \pi_{0}\right)$-transitivity. If $\underline{L} \neq(y=0)$ (if $\underline{L}=(y=c)$ then by (5.1) [4], $c \in F$ and $y=0$ is in the orbit of $y=c$ under the existing translation group of $\pi$ ) then there is an $\alpha \in F$ such that $\underline{L} I(\alpha)$ (i.e., $\underline{L} \cap \pi_{0}$ is a line of $\pi_{0}$ ). Since $y=x \alpha$ is in the line orbit of $\underline{L}$ (under the collineation group of $\pi$ ), $\pi$ is $\left((\infty), y=x \alpha, \pi_{0}\right)$ transitive. Clearly, by rechoosing $t$ to be $t+\alpha$ in $\underline{C}$ we have $\left((\infty), y=0, \pi_{0}\right)$-transitivity. And, by $(3.7)$ ( $\left.\mathrm{i}\right)$, then we have $((0)=$ $x=0, \pi_{0}$ )-transitivity (see (2.5) (5) and the remarks following (2.2)).

If $p \neq(\infty)$, there exists an $\alpha \in F$ such that $p=(\alpha)$. Rechoose $t$ in $\underline{C}$ so that $p=(0)$. If $\underline{L}=(x=0)$ we have $\left((0), x=0, \pi_{0}\right)$-transitivity and thus (see $(3.7)$ (i )) $\left((\infty), y=0, \pi_{0}\right)$-transitivity. If $\underline{L} \neq(x=0)$ we then have $\left((0), y=x(-\beta), \pi_{0}\right)$-transitivity for some $\beta \in F$. By recoordinatizing by the map $(x, y) \rightarrow\left(x+y \beta^{-1}, y\right)$ we see that $y=x(-\beta) \rightarrow$ $(x=0)$ and $(0)$ is fixed in $\pi$. Thus, we have $\left((0), x=0, \pi_{0}\right)$-transitivity.

(ii) follows from a similar argument. The proof is left to the reader.

Proposition 3.9. If $\pi$ is $\left((0,0), L_{\infty}, \pi_{0}\right)$-transitive then $\alpha a=a \alpha$ for all $a \in C$ and for all $\alpha \in F$.

Proof. It is an easy exercise to show that the collineations $(x, y)$ $\rightarrow(x, x \alpha+y) \quad \forall \alpha \in F$ and $(x, y) \rightarrow(x, y \beta) \quad \forall \beta \in F-\{0\}$ of $\pi$ induce respectively in $C$ (in $\pi$ ) automorphisms $\sigma_{\alpha}$ fixing $F$ pointwise of the form $t \sigma_{\alpha}=t+\alpha$ and collineations of the following type: $\left(t x_{1}+y_{1}, t x_{2}+y_{2}\right)$ 
$\rightarrow\left(t x_{1}+y_{1} \beta, t x_{2}+y_{2} \beta\right.$ ) for all nonzero $\beta \in F$ (note $x_{i}, y_{i} \in F, i=1,2$ ). These latter mappings fix $(0,0)$ and $(1, t) \rightarrow(\beta, t)$. Thus, $y=x t \rightarrow y$ $=x\left(\beta^{-1} t\right)$ (see (3.3)). Let $y_{1} t=t h\left(y_{1}\right)+k\left(y_{1}\right), y_{1} \in F$ and $h, k: F \rightarrow F$. Then $\left(y_{1}, y_{1} t\right) \rightarrow\left(y_{1} \beta\right.$, th $\left.\left(y_{1}\right)+k\left(y_{1}\right) \beta\right)$ and hence $\left(y_{1} \beta\right) \beta^{-1} t=t h\left(y_{1}\right)+k\left(y_{0}\right) \beta$. It follows then that $k\left(y_{1}\right) \beta=k\left(y_{1}\right)$ for all $y_{1} \in F$ and for all nonzero $\beta \in F$. Hence, $k\left(y_{1}\right)=0$ for all $y_{1} \in F$ and thus $y_{1} t=t h\left(y_{1}\right)$ for all $y_{1} \in F$ (note : $F$ cannot be $G F(2)$ ).

Now $\left(y_{1} t\right) \sigma_{\alpha}=\left(t h\left(y_{1}\right)\right) \sigma_{\alpha}$ so $y_{1}(t+\alpha)=(t+\alpha) h\left(y_{1}\right)$. By using the properties of a right vector space (see (2.4) and (3.3)) we can establish $y_{1} t+y_{1} \alpha=t h\left(y_{1}\right)+\alpha h\left(y_{1}\right)$. That is, $y_{1}=h\left(y_{1}\right)$. Therefore, $y_{1} t=t y_{1}$ for all $y_{1} \in F$.

Let $a \in C$ and $a=t \alpha_{1}+\alpha_{2} ; \alpha_{1}, \alpha_{2} \in F$. Then $a \alpha=\left(t \alpha_{1}+\alpha_{2}\right) \alpha=$ $\left(t \alpha_{1}\right) \alpha+\alpha_{2} \alpha=t\left(\alpha_{1} \alpha\right)+\alpha_{2} \alpha=\alpha\left(t \alpha_{1}\right)+\alpha \alpha_{2}=\alpha\left(t \alpha_{1}+\alpha_{2}\right)=\alpha a$ (see (3.3)). Therefore, (3.9) is proved.

Proposition 3.10. If $\pi$ is $\left((\infty), x=0, \pi_{0}\right)$-and $\left((0), x=0, \pi_{0}\right)$-transitive, then $\alpha a=\alpha \alpha$ for all $\alpha \in F$ and for all $a \in C$.

Proof. Let $*$ indicate multiplication in $\underline{\pi}$ (in $\underline{C}$ ). We will show that, $a * \alpha=\alpha * a$ in $\pi$. Fryxell [2], Lemma 2.1, shows that under these conditions, $\alpha \alpha=\alpha \alpha$ in the semi-translation plane $\pi$. (Fryxell's argument is straightforward and can be supplied by the reader by an application of (2.7) (e).)

If we derive $\pi$ we obtain $\underline{\pi}$ so that remarks that we made as to the form that collineations of $\pi$ take in $\pi$ also hold for the form collineations of $\pi$ take in $\pi$.

By (3.1), the $\left((0), x=0, \pi_{0}\right)$-collineations of $\pi$ are represented by the mappings $(x, y) \rightarrow(x \alpha, y)$. Letting $x=t x_{1}+x_{2}, y=t y_{1}+y_{2}, x_{i}, y_{i} \in F$, $i=1,2$, we clearly have the following induced collineations in $\underline{\pi}$ (see (2.6)): $\left(t x_{1}+y_{1}, t x_{2}+y_{2}\right) \rightarrow\left(t\left(x_{1} \alpha\right)+y_{1}, t\left(x_{2} \alpha\right)+y_{2}\right)$ (note that $t * \alpha=t \alpha$ by $(2.7)(d))$. By $(2.10)$, the $\left((0), x=0, \pi_{0}\right)$-collineations of $\pi$ induce an automorphism group in $\underline{\pi}$ (in $\underline{C}$ ) of order $q$-1 fixing $F$ pointwise. We see that such automorphisms are of the form $t \rightarrow t \alpha$ for all nonzero $\alpha \in F$.

Now let $\alpha * t=t f(\alpha)+G(\alpha)$ for $f(\alpha), G(\alpha) \in F$. The $\left((\infty), x=0, \pi_{0}\right)$ collineations of $\pi$ induce an automorphism group in $\underline{\pi}$ (in $\underline{C}$ ) of order $q$ fixing $F$ pointwise of the form $t \rightarrow t+\alpha$ for all $\alpha \in F$. Hence $\alpha *(t+\beta)=(t+\beta) f(\alpha)+G(\alpha)$ for $\alpha, \beta \in F$. By the properties of the right vector space and (3.2), we have $\alpha * t+\alpha \beta=t f(\alpha)+\beta f(\alpha)+G(\alpha)$ and thus $\alpha=f(\alpha)$. Therefore, $\alpha * t=t \alpha+G(\alpha)$. Using the automorphisms of the form $t \rightarrow t \beta$ we have $\alpha *(t \beta)=(t \beta) \alpha+G(\alpha)$. But, $\alpha *(t \beta)=$ $(\alpha * t) \beta=(t \alpha+G(\alpha)) \beta=t(\alpha \beta)+G(\alpha) \beta$. Hence, $G(\alpha)=G(\alpha) \beta$ for all $\alpha \in F$ and for all nonzero $\beta \in F$. Since $q>2$, we have $G(\alpha)=0$ for 
all $\alpha$ in $F$. Thus, $\alpha * t=t \alpha$. By the argument of (3.9), $\alpha \alpha=\alpha * a$ for all $\alpha \in F$ and for all $a \in \underline{C}$ (see (3.1)).

LEMMA 3.11. Let $\left(t \alpha_{1}\right)\left(t \alpha_{2}\right)=\operatorname{th}\left(\alpha_{1}, \alpha_{2}\right)+k\left(a_{1}, a_{2}\right)$ in $C ; h, k: F \rightarrow F$; $\alpha_{1}, \alpha_{2} \in F$. Then, if $\pi$ is $\left((\infty), x=0, \pi_{0}\right)$-transitive, there cannot exist functions $f$ and $g: F \rightarrow F$ such that $h\left(\alpha_{1}, \alpha_{2}\right)=f\left(\alpha_{1}\right) \alpha_{2}$ and $k\left(\alpha_{1}\right) \alpha_{2}=$ $g\left(\alpha_{1}\right) \alpha_{2}$ unless $\pi$ is the Hughes plane of order 9 (see [3], [9], or [18] for the definition of the Hughes plane).

Proof. Suppose there do exist functions $f$ and $g$ with the above properties. Since $\underline{C}$ is of dimension 2 over its kernel, it is easy to see that if $\left(t \alpha_{1}\right) *\left(t \alpha_{2}\right)=t \underline{h}\left(\alpha_{1}, \alpha_{2}\right)+\underline{k}\left({ }_{1} \alpha, \alpha_{2}\right)$ in $\underline{C} ; \underline{h}, \underline{k}: F \times F \rightarrow F$ and $\alpha_{1}, \alpha_{2} \in F$ then $\underline{h}\left(\alpha_{1}, 1\right) \alpha_{2}=\underline{h}\left(\alpha_{1}, \alpha_{2}\right)$ and $\underline{k}\left(\alpha_{1}, 1\right) \alpha_{2}=\underline{k}\left(\alpha_{1}, \alpha_{2}\right)$.

By (2.7) (e), $\left(t \alpha_{1}\right)\left(t \alpha_{2}\right)=t f\left(\alpha_{1}\right) \alpha_{2}+g\left(\alpha_{1}\right) \alpha_{2}$ if and only if $\left(t \alpha_{1}+\right.$ $\left.f\left(\alpha_{1}\right) \alpha_{2}\right) *\left(t u_{1}+u_{2}\right)=g\left(\alpha_{1}\right) \alpha_{2}$ where $\alpha_{2} *\left(t u_{1}+u_{2}\right)=t ; \alpha_{i}, u_{i} \in F-\{0\}, i=1,2$. Since $\underline{\pi}$ is $((\quad), x=0)$ - and $\left((x), y=0, \pi_{0}\right)$-transitive, $\alpha_{2} *\left(t u_{1}+u_{2}\right)=$ $\alpha_{2} *\left(t u_{1}\right)+\alpha_{2} u_{2}=\left(\alpha_{2} * t\right) u_{1}+\alpha_{2} u_{2}$ (see (3.2) and (3.4)) and by the first part of the argument of (3.10), $\alpha_{2} * t=t \alpha_{2}+G\left(\alpha_{2}\right), G\left(\alpha_{2}\right) \in F$. Thus, $t=\left(t \alpha_{2}+G\left(\alpha_{2}\right)\right) u_{1}+\alpha_{2} u_{2}=t\left(\alpha_{2} u_{2}\right)+G\left(\alpha_{2}\right) u_{1}+\alpha_{2} u_{2}$. Equating vector parts of the last equation, we have $u_{1}=\alpha_{2}^{-1}$ and $u_{2}=-G\left(\alpha_{2}\right) \alpha_{2}^{-2}$. Thus, $\left(t \alpha_{1}+f\left(\alpha_{1}\right) \alpha_{2}\right) *\left(t \alpha_{2}^{-1}-\alpha_{2}^{-2} G\left(\alpha_{2}\right)\right)=g\left(\alpha_{1}\right) \alpha_{2}$.

Now since $\pi$ is $\left((\infty), x=0, \pi_{0}\right)$-transitive, $\pi$ (and also $\pi$ ) is automorphic (i.e., $C$ and $\underline{C}$ are automorphic) (see (2.8)). Hence, by (2.9), $\left(t \alpha_{1}+f\left(\alpha_{1}\right) \alpha_{2}\right) *\left(t \alpha_{2}^{-1}-\alpha_{2}^{-2} G\left(\alpha_{2}\right)\right)=t\left[\underline{h}\left(\alpha_{1}, 1\right) \alpha_{2}^{-1}-f\left(\alpha_{1}\right)-\alpha_{1} \alpha_{2}^{-2} G\left(\alpha_{2}\right)\right]+$ $f\left(\alpha_{1}\right) \underline{h}\left(\alpha_{1}, 1\right) \alpha_{1}^{-1}+\underline{k}\left(\alpha_{1}, 1\right) \alpha_{2}^{-1}\left(f\left(\alpha_{1}\right)\right)^{2} \alpha_{1}^{-1} \alpha_{2}-f\left(\alpha_{1}\right) \alpha_{2}^{-1} G\left(\alpha_{2}\right)$.

Equating vector parts, we have :

(1) $\underline{h}\left(\alpha_{1}, 1\right) \alpha_{2}^{-1}-f\left(\alpha_{1}\right)-\alpha_{1} \alpha_{2}^{-2} G\left(\alpha_{2}\right)=0$ and

(2) $\bar{f}\left(\alpha_{1}\right) \underline{h}\left(\alpha_{1}, 1\right) \alpha_{1}^{-1}+\underline{k}\left(\alpha_{1}, 1\right) \alpha_{2}^{-1}-\left(f\left(\alpha_{1}\right)\right)^{2} \alpha_{1}^{-1} \alpha_{2}-f\left(\alpha_{1}\right) \alpha_{2}^{-1} G\left(\alpha_{2}\right)=$ $g\left(\alpha_{1}\right) \alpha_{2}$ for all nonzero $\alpha_{1}, \alpha_{2} \in F$. Let $\alpha_{2}=1$ in $(1)$, then $\underline{h}\left(\alpha_{1}, 1\right)=f\left(\alpha_{1}\right)$. Substituting $\underline{h}\left(\alpha_{1}, 1\right)=f\left(\alpha_{1}\right)$ back into $(1)$, we have:

(3) $f\left(\alpha_{1}\right)\left(\alpha_{2}-1\right)=-\alpha_{1} \alpha_{2}^{-1} G\left(\alpha_{2}\right)$. Let $f(1)=\beta$ and let $\alpha_{1}=1$ in (3). Hence, $G\left(\alpha_{2}\right)=-\beta\left(\alpha_{2}-1\right) \alpha_{2}$ so,

(4) $f\left(\alpha_{1}\right)=\alpha_{1} \beta$ for all $\alpha_{1} \in F$. So, by substituting $\underline{k}\left(\alpha_{1}, 1\right)=g\left(\alpha_{1}\right)$ (let $\alpha_{2}=1$ in (2)) and (3) and (4) into (2), we have

(5) $g\left(\alpha_{1}\right)\left(\alpha_{2}^{-1}-\alpha_{2}\right)=0$ for all nonzero $\alpha_{1}, \alpha_{2} \in F$. If $\alpha_{2}^{-1}=\alpha_{2}$ for all nonzero elements of $F$ then $F=G F$ (3). In this case, $\underline{\pi}$ is a dual translation plane of order 9. Fryxell [2] has pointed out that, in this case, $\pi$ would be the Hughes plane of order 9 . Therefore, by our assumptions, either $\pi$ is the Hughes plane of order 9 or $g\left(\alpha_{1}\right)=0$ for all nonzero $\alpha_{1} \in F$. But in the latter case, $t^{2}=t f(1)+g(1)+t \beta$ which is a contradiction to multiplication being a loop. Thus, (3.11) is proved. 
Proposition 3.12. $\pi$ cannot be both $\left((\infty), x=0, \pi_{0}\right)$ - and $((\infty), y$ $\left.=0, \pi_{0}\right)$-transitive.

Proof. If $\pi$ is so transitive then clearly (see (3.4)) if $\left(t \alpha_{1}\right)\left(t \alpha_{2}\right)=$ $t h\left(\alpha_{1}, \alpha_{2}\right)+k\left(\alpha_{1}, \alpha_{2}\right)$ then $k\left(\alpha_{1}, 1\right) \alpha_{2}=k\left(\alpha_{1}, \alpha_{2}\right)$ and $h\left(\alpha_{1}, 1\right) \alpha_{2}=h\left(\alpha_{1}, \alpha_{2}\right)$. But, this is contrary to (3.11).

THEOREM 3.13. If $\pi$ is strict, then $L_{\infty}$ is invariant under the full collineation group of the projective extension of $\pi$. In this case, the full group of $\pi$ is the inherited group (see [18] for this definition).

Proof. (Ostrom [10] gives a proof for $q>4$.) Suppose $L_{\infty}$ is moved. By Lemma 3 [10], we may take the image of $L_{\infty}$ to be $x=0$. In this situation, by Lemmas 4,5 and 6 [10], if $\left(t \alpha_{1}\right)\left(t \alpha_{2}\right)=t h\left(\alpha_{1}, \alpha_{2}\right)$ $+k\left(\alpha_{1}, \alpha_{2}\right)$ then there exist functions $f, g: F \rightarrow F$ such that $h\left(\alpha_{1}, \alpha_{2}\right)$ $=f\left(\alpha_{1}\right) \alpha_{2}$ and $k\left(\alpha_{1}, \alpha_{2}\right)=g\left(\alpha_{1}\right) \alpha_{2}$. But, this is a contradiction by (3.11).

Lemma 3.14 . $\pi$ cannot be both $\left((\infty), x=0, \pi_{0}\right)$ - and $\left((0,0), L_{\infty}, \pi_{0}\right)$ transitive.

Proof. If $\pi$ is so transitive, we have pointed out in (3.11) that both $\pi$ and $\pi$ (i.e., $\underline{C}$ and $C$ ) are automorphic. By (3.9) and Lemma 2.1 [2], elements of $F$ commute with elements of $C$ and of $\underline{C}$. Hence, by (3.3), $\pi$ admits collineations of the form $(x, y) \rightarrow(x \alpha, y \alpha)$ for all nonzero $\alpha \in F$. By (2.6), it is easy to see that $\underline{\pi}$ also admits collineations of this same form. Recall $\underline{\pi}$ is $\left((\infty), y=0, \bar{\pi}_{0}\right)$-transitive, so (see 3.4)) $\underline{\pi}$ admits collineations of the form $(\underline{x}, \underline{y}) \rightarrow(\underline{x}, \underline{y} \beta)$ for all nonzero $\beta \in F$. Hence, $(\underline{x}, \underline{y}) \rightarrow(\underline{x} \alpha, \underline{y} \alpha)$ followed by $(\underline{x}, \underline{y}) \rightarrow\left(\underline{x}, \underline{y} \alpha^{-1}\right)$ will yield collineations represented by $(\underline{x}, \underline{y}) \rightarrow(\underline{x} \alpha, \underline{y})$. By (3.3), (3.4), and (3.5), we have that elements of $F$ associate and commute with the elements of $\underline{C}$. By Theorem 5 [8], we know that since $\underline{C}$ is automorphic, $q \leqq 3$. However, we have assumed that $q>3$ and thus we have a contradiction.

Lemma $3.15 . \quad \pi$ cannot be both $\left((0), x=0, \pi_{0}\right)$ - and $\left((0,0), L_{\infty}, \pi_{0}\right)$ transitive.

Proof. Suppose $\pi$ is so transitive. By the argument of (3.14) the elements of $F$ associate and commute with the elements of $\underline{C}$ in ㅈ. By our assumptions we see (by the argument of (3.10)) that there is an automorphism group of $\underline{C}$ of order $q-1$ which fixes $F$ pointwise and is represented by $t \rightarrow t \alpha$ for all nonzero $\alpha \in F$.

Let $\left(t \alpha_{1}\right) *\left(t \alpha_{2}\right)=t \underline{h}\left(\alpha_{1}, \alpha_{2}\right)+\underline{k}\left(\alpha_{1}, \alpha_{2}\right)$ in $\underline{C}$ for functions $\underline{h}, k: F \times$ 
$F \rightarrow F$. Thus, $t^{2}=t \underline{h}(1,1)+\underline{k}(1,1)$. Applying the automorphism $t \rightarrow t \alpha$, we have $(t \alpha)^{2}=(t \alpha) \underline{h}(1,1)+\underline{k}(1,1)$. But, $(t \alpha)^{2}=t^{2} \alpha^{2}=t \underline{h}(1,1) \alpha^{2}+$ $\underline{k}(1,1) \alpha^{2}$, so by equating vector parts of the two expressions for $t^{2}$ we have : $\underline{h}(1,1) \alpha^{-1}=\underline{h}(1,1)$ and $\underline{k}(1,1) \alpha^{-2}=\underline{k}(1,1)$ for all nonzero $\alpha \in F$. But, since $q>3$ this implies $\underline{k}(1,1)=\underline{h}(1,1)=0$ and thus that $t^{2}=0$. But this is a contradiction to multiplication being a loop.

Lemma 3.16 . $\pi$ cannot be both $\left((0), x=0, \pi_{0}\right)$ - and $\left((\infty), y=0, \pi_{0}\right)$ transitive. $F=G F(4)$ or $F=G F(5)$ may be exceptions.

Proof. Suppose $\pi$ is so transitive. By (3.1) and (3.4) $c(\alpha m)=$ $(c \alpha) m$ and $(\mathrm{cm}) \alpha=c(m \alpha)$ for all $c, m \in C$ and for all $\alpha \in F$. Moreover, $(x, y) \rightarrow(x \alpha, y \beta)$ for all $\alpha, \beta \in F-\{0\}$ represent collineations in $\pi$.

Also, $\underline{\pi}$ is $\left((\infty), x=0, \pi_{0}\right)$ - and $\left((\infty), \mathrm{y}=0, \pi_{0}\right)$-transitive, so $C$ admits automorphisms represented by $t \rightarrow t+\alpha$ for all $\alpha \in F$ which fixes $F$ elementwise and $\pi$ admits collineations represented by the mappings :

$$
\left(t x_{1}+y_{1}, t x_{2}+y_{2}\right) \stackrel{m_{\beta}}{\longrightarrow}\left(t x_{1}+y_{1} \beta, t x_{2}+y_{2} \beta\right)
$$

for all $\beta \in F-\{0\}$ (see (3.9)). Since

$$
(0,0) \stackrel{m_{\beta}}{\longrightarrow}(0,0), \quad(1, t) \stackrel{m_{\beta}}{\longrightarrow}(\beta, t)
$$

then

$$
y=x t \stackrel{m_{3}}{\longrightarrow} y=x\left(\beta^{-1} t\right) .
$$

Let $y_{1} t=t h\left(y_{1}\right)+k\left(y_{1}\right), y_{1} \in F, h, k: F \rightarrow F$. Then

$$
\left(y_{1}, y_{1} t\right) \stackrel{m_{\beta}}{\longrightarrow}\left(y_{1} \beta, \operatorname{th}\left(y_{1}\right)+h\left(y_{1}\right) \beta\right)
$$

and hence $\left(y_{1} \beta\right) \beta^{-1} t=t h\left(y_{1}\right)+k\left(y_{1}\right) \beta$. Thus, $k\left(y_{1}\right) \beta=k\left(y_{1}\right)$ for $y_{1} \in F$ and for all nonzero $\beta \in F$. Hence, $k\left(y_{1}\right)=0$ for all $y_{1} \in F$.

Since $(\alpha \beta) t=\alpha(\beta t) \forall \alpha, \beta \in F$, it follows that $h(\alpha \beta)=h(\alpha) h(\beta)$. Moreover, $h$ is clearly one-to-one (and hence onto) so $h$ is a multiplication automorphism of $F-\{0\}$.

It is easy to see that $\alpha * t=t h^{-1}(\alpha)$ (see (2.7)(e)) where $*$ denotes multiplication in $\underline{C}$.

By the remarks following (2.6), the collineations $(x, y) \rightarrow(2 x, y \beta)$, $\alpha, \beta \in F-\{0\}$ of $\pi$ induce collineations in $\underline{\pi}$ of the following form:

$$
\left(t x_{1}+x_{2}, t y_{1}+y_{2}\right) \stackrel{\chi_{x, \beta}}{\longrightarrow}\left(t\left(x_{1} \alpha\right)+\left(x_{2} \beta\right), t\left(y_{1} \alpha\right)+\left(y_{2} \beta\right)\right)
$$

for all $\alpha, \beta \in F-\{0\}$ for $x_{i}, y_{i} \in F, i=1,2$. 
Now $\left(1, t m_{1}+m_{2}\right) \stackrel{\chi_{\alpha, \beta}}{\longrightarrow}\left(\beta, t\left(m_{1} \alpha\right)+m_{2} \beta\right), \quad m_{i} \in F, \quad i=1,2, \quad$ and $(0,0) \stackrel{\chi_{\alpha, \beta}}{\longrightarrow}(0,0)$. Therefore

$$
\begin{aligned}
y=x *\left(t m_{1}+m_{2}\right) \stackrel{\chi_{\alpha, \beta}}{\longrightarrow} y & =x *\left(t \bar{m}_{1}+\bar{m}_{2}\right) \ni \beta *\left(t \bar{m}_{1}+\bar{m}_{2}\right) \\
& =t\left(m_{1} \alpha\right)+m_{2} \beta .
\end{aligned}
$$

Since we have the full left distributive law in $\underline{C}$ it follows that

$$
t\left(h^{-1}(\beta) \bar{m}_{1}\right)+\beta \bar{m}_{2}=t\left(m_{1} \alpha\right)+m_{2} \beta
$$

and hence $\bar{m}_{1}=m_{1} \alpha h^{-1}\left(\beta^{-1}\right), \bar{m}_{2}=m_{2}$. Thus,

$$
y=x *\left(t m_{1}+m_{2}\right) \longrightarrow y=x *\left(t\left(m_{1} \alpha h^{-1}\left(\beta^{-1}\right)\right)+m_{2}\right) .
$$

Let $(t \delta+\gamma) * t=t f(\delta, \gamma)+g(\delta, \gamma)$ for all $\delta, \gamma \in F$ where $f, g: F \times F \rightarrow F$. Then $(t \delta+\gamma) *\left(t m_{1}+m_{2}\right)=t\left(f(\delta, \gamma) m_{1}+\delta m_{2}\right)+\left(g(\delta, \gamma) m_{1}+\gamma m_{2}\right)$. So.

$$
\begin{aligned}
&\left(t \delta+\gamma, t\left(f(\delta, \gamma) m_{1}+\delta m_{2}\right)\right)+ \\
&\left(g(\delta, \gamma) m_{1}+\gamma m_{2}\right) \stackrel{\chi_{a, \beta}}{\longrightarrow}\left(t(\delta \alpha)+\gamma \beta, t\left(f(\delta, \gamma) m_{1}+\delta m_{2}\right) \alpha+\right. \\
&\left.\left(g(\delta, \gamma) m_{1}+\gamma m_{2}\right) \beta\right) .
\end{aligned}
$$

Hence,

$(t(\delta \alpha)+\gamma \beta) *\left(t\left(m_{1} \alpha h^{-1}\left(\beta^{-1}\right)+m_{2}\right)=\left(t\left(f(\delta, \gamma) m_{1}+\delta m_{2}\right) \alpha+\left(g(\delta, \gamma) m_{1}+\gamma m_{2}\right) \beta \ldots\right.\right.$

But,

$$
\begin{aligned}
(t(\delta \alpha)+\gamma \beta) *\left(t\left(m_{1} \alpha h^{-1}\left(\beta^{-1}\right)+m_{2}\right)=\right. & t\left(f(\delta \alpha, \gamma \beta) m_{1} \alpha h^{-1}\left(\beta^{-1}\right)+\delta \alpha m_{2}\right)+ \\
& \left(g(\delta \alpha, \gamma \beta) m_{1} \alpha h^{-1}\left(\beta^{-1}\right)+\gamma \beta m_{2}\right) .
\end{aligned}
$$

Equating vector parts of the last two equations,

$$
f(\delta \alpha, \gamma \beta) m_{1} \alpha h^{-1}\left(\beta^{-1}\right)+\delta \alpha m_{2}=\left(f(\delta, \gamma) m_{1}+\delta m_{2}\right) \alpha
$$

and

$$
g(\delta \alpha, \gamma \beta) m_{1} \alpha h^{-1}\left(\beta^{-1}\right)+\gamma \beta m_{2}=\left(g(\delta, \gamma) m_{1}+\gamma m_{2}\right) \beta .
$$

For $m_{1} \neq 0$, we have:

$$
f(\delta \alpha, \gamma \beta) h^{-1}\left(\beta^{-1}\right)=f(\delta, \gamma)
$$

and

$$
g(\delta \alpha, \gamma \beta) h^{-1}\left(\beta^{-1}\right)=g(\delta, \gamma) \beta \alpha^{-1}
$$

for all $\alpha, \beta \in F$ such that $\alpha \beta \neq 0$ and for all $\delta, \gamma \in F$. Let $t^{2}=t \theta+\rho$, for some $\theta, \rho \in F$ (clearly $\rho \neq 0$ ). Then

$$
(t, t \theta+\rho) \in(y=x * t) \stackrel{\chi_{1, \beta}}{\longrightarrow}(t, t \theta+\rho \beta) \in\left(y=x *\left(t h^{-1}\left(\beta^{-1}\right)\right)\right.
$$


so that $t *\left(t h^{-1}\left(\beta^{-1}\right)\right)=t \theta+\rho \beta$. But,

$$
t *\left(t h^{-1}\left(\beta^{-1}\right)\right)=t^{2} h^{-1}\left(\beta^{-1}\right)=(t \theta+\rho) h^{-1}\left(\beta^{-1}\right)=t\left(\theta h^{-1}\left(\beta^{-1}\right)\right)+\rho h^{-1}\left(\beta^{-1}\right) .
$$

Hence, $\theta=\theta h^{-1}\left(\beta^{-1}\right)$ and $\rho h^{-1}\left(\beta^{-1}\right)=\rho \beta$. Since $\rho \neq 0$, we have $h^{-1}\left(\beta^{-1}\right)=\beta$ for all $\beta \in F-\{0\}$ and $\theta=0$. So $h=h^{-1}$ and $f(\delta, \gamma)=$ $f(\delta \alpha, \gamma \beta) \beta$ and $g(\delta, \gamma)=g(\delta \alpha, \gamma \beta) \alpha$ for all nonzero $a, \beta \in F$ and for all $\hat{o}, \gamma \in F$. Letting $\hat{o}=\alpha^{-1}$ and $\gamma=\beta^{-1}$ we obtain $f\left(\alpha^{-1}, \beta^{-1}\right)=f(1,1) \beta$ and $g\left(\alpha^{-1}, \beta^{-1}\right)=g(1,1) \alpha$. Let $f(1,1)=f$ and $g(1,1)=g$. Thus,

$$
\begin{aligned}
& f(\alpha, \beta)=f \beta^{-1}, \alpha \beta \neq 0 \\
& f(0, \beta)=\beta^{-1}, \beta \neq 0 \\
& f(\alpha, 0)=0
\end{aligned}
$$

and

$$
\begin{aligned}
& g(\alpha, \beta)=g \alpha^{-1}, \alpha \beta \neq 0 \\
& g(0, \beta)=0 \\
& g(\alpha, 0)=\rho \alpha^{-1} \text { where } t^{2}=\rho .
\end{aligned}
$$

(Recall $t^{2}=\rho$ and $\underline{C}$ admits automorphisms of the form $t \rightarrow t \alpha$ which fix $F$ elementwise. Hence $(t \alpha) *(t \alpha)=\rho$ which implies $(t \alpha) * t=\rho \alpha^{-1}$.)

Now suppose that we have at least seven elements in $F$, choose $\gamma \neq 0,1, \rho^{-1}$ or $(f+g)^{-1}$ if $(f+g) \neq 0$. Now $(t \alpha+\beta) *(t+\gamma)=t+1$ must have a unique solution for $t \alpha+\beta$. If $\alpha=0$ then $t \beta^{-1}+\beta \gamma=t+1$ which implies $\gamma=1$, contrary to assumption. If $\beta=0$ then $\left(t(\alpha \gamma)+\alpha^{-1} \rho\right)$ $=t+1$ which implies that $\gamma=\rho^{-1}$, again contrary to assumption. Thus, if we are to have a solution, $\alpha \beta \neq 0$. In this case, $(t \alpha+\beta) *$ $(t+\gamma)=t\left(f \beta^{-1}+\alpha \gamma\right)+\left(g \alpha^{-1}+\beta \gamma\right)=t+1$. So $\beta^{-1} f+\alpha \gamma=1$ and $\alpha^{-1} g+\beta \gamma=1$. These last equations are equivalent to $f+\alpha \gamma \beta=\beta$ and $g+\alpha \gamma \beta=\alpha$. If these equations have a solution $(\alpha, \beta)$ then $\left(\left(\gamma^{-1}-\beta\right),\left(\gamma^{-1}-\alpha\right)\right)$ is also a solution. That is,

$$
\begin{aligned}
f+\left(\gamma^{-1}-\beta\right) \gamma\left(\gamma^{-1}-\alpha\right) & =f+\gamma^{-1}-(\alpha+\beta)+\alpha \gamma \beta \\
& =f+\alpha \gamma \beta+\gamma^{-1}-(\alpha+\beta) \\
& =\gamma^{-1}-\alpha .
\end{aligned}
$$

Also, $g \div\left(\gamma^{-1}-\beta\right) \gamma\left(\gamma^{-1}-\alpha\right)=\gamma^{-1}-\beta$. Thus, in order to insure that we have a unique solution it follows that $\gamma^{-1}-\beta=\alpha$ and $\gamma^{-1}-\alpha=\beta$ so that $\gamma^{-1}=\alpha+\beta$. If the characteristic of $F$ is 2 , then $f+\alpha \beta \gamma=\beta$ and $g+\alpha \beta \gamma=\alpha$ implies that $f+g=\alpha+\beta=\gamma^{-1}$, contrary to the choice of $\gamma$. Thus, char. $F$ is odd and the equations $f+\alpha \beta \gamma=\beta$ and $g+\alpha \beta \gamma=\alpha$ are equivalent to $2 f+2 \alpha \beta \gamma=2 \beta$ and $2 g+2 \alpha \beta \gamma=2 \gamma$. Since $f+g+2 \alpha \beta \gamma=\alpha+\beta=\gamma^{-1}$ we have $f+g-2 f=\gamma^{-1}-2 \beta$ or $f-g+\gamma^{-1}=2 \beta$. Similarly, $g-f+\gamma^{-1}=2 \alpha$. 
Now $\left.\begin{array}{l}f+\alpha \beta \gamma=\beta \\ g+\alpha \beta \gamma=\alpha\end{array}\right\}$ if and only if $\left\{\begin{array}{l}4 f+(2 \alpha)(2 \beta) \gamma=4 \beta \\ 4 g+(2 \alpha)(2 \beta) \gamma=4 \alpha .\end{array}\right.$

If a unique solution $(\alpha, \beta)$ exists, it must be $\left(\left(g-f+\gamma^{-1}\right) 2^{-1}\right.$, $\left.\left(f-g+\gamma^{-1}\right) 2^{-1}\right)$ and must satisfy the latter equations. Hence

$$
4 f+\left(g-f+\gamma^{-1}\right)\left(f-g+\gamma^{-1}\right) \gamma=2\left(f-g+\gamma^{-1}\right)
$$

if and only if $2(f+g)-\gamma(g-f)^{2}=\gamma^{-1}$. Thus, if $x(t+\gamma)=t+1$ is to have a unique solution for $x$ and $\gamma \neq 0,1, \rho^{-1},(f+g)^{-1}$ if $f \neq-g$ then $\gamma$ must satisfy the equation $2(f+g)-\gamma(g-f)^{2}=\gamma^{-1}$. If $\gamma$ can take on other values, say $w$ and $\Sigma$ distinct from $0,1, \rho^{-1}$, $(f+g)^{-1}$ then $\gamma(g-f)^{2}+\gamma^{-1}=w(g-f)^{2}+w^{-1}=\Sigma(g-f)^{2}+\Sigma^{-1}$. Thus, $(\gamma-w)(g-f)^{2}=w^{-1}-\gamma^{-1}=w^{-1} \gamma^{-1}(\gamma-w)$ which implies that $(g-f)^{2}=w^{-1} \gamma^{-1}$. It follows that we have $w^{-1} \gamma^{-1}=\Sigma^{-1} \gamma^{-1}$ so that $w^{-1}=\Sigma^{-1}$, contrary to assumption. Thus, we have a contradiction to the initial assumption and (3.16) is proved. Note that we may have possible exceptions if $F=G F(4)$ or $G F(5)$.

The proof of the following lemma is routine and is left to the reader.

LEMMA 3.17. Each $\left((0), y=0, \pi_{0}\right)$-collineation is represented by a mapping of the form $(x, y) \rightarrow(x+y \alpha, y)$ for some $\alpha \in F$.

Lemma 3.18. $\pi$ cannot be both $\left((\infty), x=0, \pi_{0}\right)$ - and $\left((0), y=0, \pi_{0}\right)$ transitive.

Proof. Suppose $\pi$ is so transitive. By (3.17), we have collineations in $\pi$ represented by $(x, y) \rightarrow(x+y \alpha, y)$. Therefore, in $\underline{\pi}$ we have collineations: $\forall \alpha \in F$,

$$
\left(t x_{1}+x_{2}, t y_{1}+y_{2}\right) \longrightarrow\left(t\left(x_{1}+x_{2} \alpha\right)+x_{2}, t\left(y_{1}+y_{2} \alpha\right)+y_{2}\right)
$$

so

$$
\begin{gathered}
(1, t) \cup(0,0) \longrightarrow(t \alpha+1, t) \cup(0,0) \\
\therefore y=x t \longrightarrow y=x\left(t m_{1}+m_{2}\right)
\end{gathered}
$$

such that $(t \alpha+1)\left(t m_{1}+m_{2}\right)=t . \quad \underline{C}$ is automorphic since $\pi$ is: $\left((\infty), x=0, \pi_{0}\right)$-transitive.

$$
\begin{aligned}
\therefore \quad(t \alpha+1)\left(t m_{1}\right. & \left.+m_{2}\right)=t\left[(h(\alpha, 1)-1) m_{1}+\alpha m_{2}\right] \\
& +\left(\alpha^{-1} h(\alpha, 1)+\mathrm{k}(\alpha, 1)-\alpha^{-1}\right) m_{1} \div m_{2}
\end{aligned}
$$

where

$$
(t \alpha)\left(t m_{1}\right)=\operatorname{th}\left(\alpha, m_{1}\right)+k\left(\alpha, m_{\mathrm{i}}\right)=(\operatorname{th}(\alpha, 1)+k(\alpha, 1)) m:
$$

for $h, k: F \times F \rightarrow F$. (Note the multiplication denoted by juxtaposi- 
tion is now assumed to be that of $\underline{C}$.)

$$
\begin{aligned}
& \therefore \quad(1) \quad(h(\alpha, 1)-1) m_{1}+\alpha m_{2}=1 \quad \text { and } \\
&(2)\left(\alpha^{-1} h(\alpha, 1)+k(\alpha, 1)-\alpha^{-1}\right) m_{1}+m_{2}=0 .
\end{aligned}
$$

Solving for $m_{1}$ and $m_{2}$, we have :

(3) $m_{1}=(-\alpha k(\alpha, 1))^{-1} \quad$ and

$$
\text { (4) } m_{2}=a^{-1}\left(1+(h(\alpha, 1)-1) k(\alpha, 1)^{-1} \alpha^{-1}\right) \text {. }
$$

Now

$$
\left(t, t^{2}=t h(1,1)+k(1,1)\right) \longrightarrow(t, t(h(1,1)+k(1,1) \alpha)+k(1,1))
$$

and

$$
\begin{aligned}
y=x t & \longrightarrow y=x\left(t m_{1}+m_{2}\right) . \\
\left(t, t^{2}\right) \in y=x t \text { so } t\left(t m_{1}+\right. & \left.m_{2}\right)=t(h(1,1)+k(1,1) \alpha)+k(1,1) . \quad \text { Also } \\
t\left(t m_{1}+m_{2}\right) & =t\left(t m_{1}\right)+t m_{2}=t^{2} m_{1}+t m_{2} \\
& =\left(t h(1,1)+k(1,1) m_{1}+t m_{2}\right. \\
& =t\left(h(1,1) m_{1}+m_{2}\right)+k(1,1) m_{1} .
\end{aligned}
$$

Equating vector components we have $k(1,1) m_{1}=k(1,1)$ and

$$
h(1,1) m_{1}+m_{2}=h(1,1)+k(1,1) \alpha .
$$

From the first equation (since $k(1,1) \neq 0$ ) we have

$$
\text { (5) } m_{1}=1 \text { and thus } k(\alpha, 1)=-\alpha^{-1} \text {. }
$$

Also, $h(1,1) m_{1}+m_{2}=h(1,1)+k(1,1) \alpha$ implies $k(1,1) \alpha=m_{2}$. By !(5), $k(1,1) \alpha=-\alpha$. Hence,

$$
\text { (6) } m_{2}=-\alpha \text { and (see (1)) } h(\alpha, 1)=2+\alpha^{2} \text {. }
$$

Therefore, $y=x t \rightarrow y=x(t-\alpha)$.

Let $\beta t=t f(\beta)+g(\beta)$ in $\underline{C}$ for $f, g: F \rightarrow F$. Since $\pi$ is $\left((\infty), x=0, \pi_{0}\right)$-transitive, $c(\alpha+\bar{m})=c \alpha+c m$ for all $c, m \in \underline{C}$ and for all $\alpha \in F$ (see the proof of (3.2)). Applying the automorphisms $t \rightarrow t+\alpha$ which fix $F$ elementwise,

$$
\beta(t+\alpha)=(t+\alpha) f(\beta)+g(\beta)=t f(\beta)+\alpha f(\beta)+g(\beta) .
$$

Since $\beta(t+\alpha)=\beta t+\beta \alpha$, it follows that $\alpha f(\beta)=\beta \alpha$ so that $f(\beta)=\beta$.

Thus,

$$
(\beta, \beta t=t \beta+g(\beta)) \longrightarrow(t(\beta \alpha)+\beta, t(\beta+g(\beta) \alpha)+g(\beta))
$$


and hence $(t(\beta \alpha)+\beta)(t-\alpha)=t(\beta+g(\beta) \alpha)+g(\beta)$.

$$
\begin{aligned}
(t(\beta \alpha)+\beta)(t-\alpha)=t[h(\beta \alpha, 1)-\beta & -(\beta \alpha) \alpha]+\beta(\beta \alpha)^{-1} h(\beta \alpha, 1) \\
& +k(\beta \alpha, 1)-\beta^{2}(\beta \alpha)^{-1}-\beta \alpha .
\end{aligned}
$$

Applying (5) and (6),

$$
\begin{aligned}
(t(\beta \alpha) & +\beta)(t-\alpha)=t\left[2+(\beta \alpha)^{2}-\beta-(\beta \alpha) \alpha\right] \\
& +\beta(\beta \alpha)^{-1}\left(2+(\beta \alpha)^{2}\right)-(\beta \alpha)^{-1}-\beta^{2}(\beta \alpha)^{-1}-\beta \alpha .
\end{aligned}
$$

Thus, equating vector parts,

$$
2+(\beta \alpha)^{2}-\beta-\beta \alpha^{2}=\beta+g(\beta) \alpha
$$

and $\left.\beta(\beta \alpha)^{-1}(2+\beta \alpha)^{2}\right)-(\beta \alpha)^{-1}-\beta^{2}(\beta \alpha)^{-1}-\beta \alpha=g(\beta)$. Therefore, we have

$$
\text { (7) } g(\beta)=2 \alpha^{-1}+\beta^{2} \alpha-\beta \alpha^{-1}-\beta \alpha-\beta \alpha^{-1}
$$

and

$$
\text { (8) } g(\beta)=2 \alpha^{-1}+\beta^{2} \alpha-(\beta \alpha)^{-1}-\beta \alpha^{-1}-\beta \alpha \text {. }
$$

Equating (7) and (8), we have:

$$
(\beta \alpha)^{-1}=\beta \alpha^{-1} \text { which implies } \beta^{-1}=\beta
$$

for all nonzero in $F$. Hence, $\beta^{2}=1$ for all $\beta \in F-\{0\}$ so that $F=$ $G F(3)$. But this is contrary to our assumption. Hence (3.18) is proved.

Let $S(\pi)$ denote the set of point-line pairs $(p, L)$ such that the plane $\pi$ is $\left(p, L, \pi_{0}\right)$-transitive. In [4] we have separated semi-translation planes into types depending on the extent of $\left(p, L, \pi_{0}\right)$-transitivity the planes admit (see (3.20)). A type $T_{i}$ is a certain set of point-line pairs $(p, L)$. We shall say that a plane $\pi$ is of type $T_{i}$ if and only if $S(\pi)$ is exactly equal to the set that defines $T_{i}$. In this case, we shall use the notation $S\left(T_{i}\right)=S(\pi)$.

Definition 3.19. A plane of type $T_{1}$ will be said to be above a plane of type $T_{2}$ if and only if $S\left(T_{2}\right) \subset S\left(T_{1}\right)$.

THEOREM (Johnson [4]) 3.20. If $\pi$ is a semi-translation plane of order $q^{2}, q \neq 5$ or 9 (or its dual) then $\pi$ is of one and only one of the following types:

1-1a $\left(p, L_{\infty}\right) \in S(\pi)$ for all points $p \in L_{\infty} \cap \pi_{0}$.

1-2a $S(1-2 \mathrm{a})=S(1-1 \mathrm{a}) \cup\left\{\left(p_{\infty}, L\right)\right.$ for all lines $L$ of $\pi_{0}$ such that $L$ I $p_{\infty}$ and $p_{\infty}$ a fixed point of $\left.L_{\infty}\right\}$.

1-3a $S(1-3 \mathrm{a})=S(1-2 \mathrm{a}) \cup\left\{(p, L)\right.$ for all points $p \in L_{\infty} \cap \pi_{0}$ for all lines $L$ of $\pi_{0}$ incident with $\left.p_{\infty}\right\}$. 
$1-4 \mathrm{a} \quad S(1-4 \mathrm{a})=S(1-1 \mathrm{a}) \cup\left\{\left(p^{\prime}, L\right)\right.$ for all lines $L$ of $\pi_{0}$ such that $L$ I $k, p^{\prime} \notin L, k$ and $\left.p^{\prime} \in L_{\infty} \cap \pi_{0}\right\}$.

$1-5 \mathrm{a} \quad S(1-5 \mathrm{a})=S(1-4 \mathrm{a}) \cup\left\{(k, L)\right.$ for all lines $L$ of $\pi_{0}$ such that $L$ I $p^{\prime}, k \notin L, k, p^{\prime} \in L_{\infty} \cap \pi_{0}$.

$\begin{array}{ll}1-1 \mathrm{~b} & S(1-1 \mathrm{~b})=S(1-1 \mathrm{a}) \cup\left\{\left(p, L_{\infty}\right) \text { for all } p \in \pi_{0}-L_{\infty}\right\} . \\ 1-2 \mathrm{~b} & S(1-2 \mathrm{~b})=S(1-1 \mathrm{~b}) \cup S(1-2 \mathrm{a}) . \\ 1-3 \mathrm{~b} & S(1-3 \mathrm{~b})=S(1-1 \mathrm{~b}) \cup S(1-3 \mathrm{a}) . \\ 1-4 \mathrm{~b} & S(1-4 \mathrm{~b})=S(1-1 \mathrm{~b}) \cup S(1-4 \mathrm{a}) . \\ 1-5 \mathrm{~b} & S(1-5 \mathrm{~b})=S(1-1 \mathrm{~b}) \cup \mathrm{S}(1-5 \mathrm{a}) . \\ 1-2 \mathrm{c} & S(1-2 \mathrm{c})=S(1-2 \mathrm{~b}) \cup\left\{\left(p_{\infty}, L\right) \text { for all lines } L \text { of } \pi_{0}\right\} . \\ 1-3 \mathrm{c} & S(1-3 \mathrm{c})=S(1-3 \mathrm{~b}) \cup S(1-2 \mathrm{c}) . \\ 2-1 \mathrm{a} & S(2-1 \mathrm{a})=\left\{(p, L) \text { for all incident point-line pairs of } \pi_{0} \text { such }\right.\end{array}$ that $L$ I $p_{\infty}$ for some point $\left.p_{\infty} \in L_{\infty}\right\}$.

2-2a $S(2-2 \mathrm{a})=S(2-1 \mathrm{a}) \cup\left\{\left(p_{\infty}, L\right)\right.$ for all lines $L$ of $\pi_{0}$ such that $\left.L I p_{\infty}\right\}$.

3-1 $S(3-1)=\left\{(p, L)\right.$ for all incident point-line pairs of $\left.\pi_{0}\right\}$.

3-2 $S(3-2)=\left\{(p, L)\right.$ for all point-line pairs of $\left.\pi_{0}\right\}$.

D1-1a, D1-4a, D1-5a, D1-ib, $i=1,2,3,4,5, \mathrm{D} 2-\mathrm{ja}, \mathrm{j}=1,2=$ the duals of the above corresponding classes.

LEMMA 3.21. $\pi$ cannot be above type D1-1b. In this case then, $\pi$ cannot be of type D1-1b, D1-2b, D1-3b, D1-4b, D1-5b, D2-2a.

Proof. If $\pi$ is above D1-1b, we may choose coordinates (see (3.8)) so that $\pi$ is $\left((\infty), x=0, \pi_{0}\right)$ - and $\left((\propto), y=0, \pi_{0}\right)$-transitive. But this is contrary to (3.12). Thus, (3.21) is proved.

Lemma 3.22. If $q>5, \pi$ cannot bs above type 1-5a. Thus, $\pi$ cannot be of type 1-5a, 1-5b or 1-3c.

Proof. Suppose $\pi$ is above type $1-5$ a and is $\left(p^{\prime}, l^{\prime}, \pi_{0}\right)$-and $\left(k, \bar{l}, \pi_{0}\right)$ transitive where $k I l^{\prime}$ and $p^{\prime} I \bar{l}$. By (3.8)(i) we can choose coordinates so that $p^{\prime}=(\infty)$ and $l^{\prime}=(y=0)$. Since $k I l^{\prime}, p^{\prime} I \bar{l}$, then $k=(0)$ and $\bar{l}$ is $(x=c)$ for some $c \in C$. Hence, $\pi$ is $\left((0), x=c, \pi_{0}\right)$-transitive. By (5.1) [4], $c \in F$ and $(x=0)$ is in the orbit of $x=c$ under the existing translation group. Therefore, $\pi$ is $\left((0), x=0, \pi_{0}\right)$ - and $\left((\infty), y=0, \pi_{0}\right)$-transitive. But this is contrary to (3.16).

In the cases D1-1a, D1-4a or D1-5a, $\pi$ is a dual semi-translation plane but not a semi-translation plane. This is contrary to the corollary to Theorem 7 [15]. Hence $\pi$ cannot be of any of these types.

Lemma 3.23. $\pi$ cannot be above type 1-2b. Thus, $\pi$ cannot be of type 1-2b, 1-3b or 1-2c. 
Proof. We may choose $p_{\infty}=(\infty)$ (see (3.20) and 3.8)) so that $\pi$ is $\left.((\infty), x=0), \pi_{0}\right)$ - and $\left.(0,0), L_{\infty}, \pi_{0}\right)$-transitive. But this is a contradication by (3.14).

LEMMA 3.24. $\pi$ cannot be above type $1-4 \mathrm{~b}$.

Proof. Suppose $\pi$ is above type 1-4b. Then, letting $p^{\prime}=(0)$ and $k=(\propto)$ (see (3.20) and (3.8)), we have $\left((0), x=0, \pi_{0}\right)$ - and $\left((0,0), L_{\infty}, \pi_{0}\right)-$ transitivity. This is a contradiction by (3.15).

By (3.13), $\pi$ cannot be of type $2-1 \mathrm{a}, 2-2 \mathrm{a}, 3.1$ or $3-2$. By (3.18), $\pi$ cannot be both $\left((>), x=0, \pi_{0}\right)$ - and $\left((0), y=0, \pi_{0}\right)$-transitive. Hence, $\pi$ cannot be of type D2-1a or D2-2a (see (3.20)).

The previous lemmas and remarks establish the following theorem for planes of order $q^{2}, q>5$.

THEOREM 3.25. If $\pi$ is a strict semi-translation plane that is derived from a dual translation plane whose coordinate system is a 2-dimensional vector space over its kernel, then $\pi$ is of one and only one of the following types: 1-1a, 1-2a, 1-3a, 1-4a or 1-1b.

Note. The possible exception for $q=9$ in [4] is excluded here since $\pi_{0}$ is Desarguesian.

We have stated our propositions in terms of a coordinate system. But, given a derivable semi-translation plane $\pi$ with respect to line $L$, we may choose $L=L_{\infty}$ and the set of points of the subplane $\pi_{0}$ of $\pi$ to be the same as the set of points of $\underline{x}=0$ in $\underline{\pi}$. And, in general, choose the coordinate system as in (2.5) and (2.6). Thus, our results could be stated for an arbitrary semi-translation plane.

We have given examples of planes of each of the types 1-1a, 1-2a, 1-3a, 1-4a and 1-1b in [5].

For planes of type 1-3a and 1-1b, (3.9) and (3.10) show that $\alpha a=\alpha \alpha$ for all $\alpha \in F$ and for all $a \in C$. Furthermore, this shows if $\alpha * a \neq a * \alpha$ for some $\alpha$ in $F$ and for some $a \in \underline{C}$ (the coordinate system for the dual translation plane) then $\pi$ must be of type 1-1a, 1-2a or 1-4a. If $\pi$ is a nearfield dual translation plane of order $q^{2}$ and $q>5$, we have shown that $\pi$ is of type 1-1a if there exists an element $a \in \underline{C}$ such that $a * \alpha \neq \alpha * a$ for some $\alpha \in F$ and of type 1-1b otherwise.

The research discussed here was directed by Professor T. G. Ostrom at Washington State University and this article is based on the author's $\mathrm{Ph}$. D. dissertation. The author would like to express 
his appreciation to Professor Ostrom for suggesting the problem considered here and also for his encouragement and guidance.

\section{REFERENCES}

1. A. A. Albert, The finite planes of Ostrom, Bol. Soc. Math. Mex. (1967), 1-13.

2. R. Fryxell, Sequences of planes constructed from nearfield planes of square order, Ph. D. dissertation, Washington State University, 1964.

3. D. R. Hughes, A class of non-Desarguesian projective planes, Canad. J. Math. 9 (1957), 378-388.

4. N. L. Johnson, A classification of semi-translation planes, Canad. J. Math. (to appear)

5. — Derivable chains of planes, Bol. Un. Math. Ital. (to appear)

6. - Nonstrict semi-translation planes, Arch. Math. 20 (1969), 301-310

7. H. Lüneburg, Über projektive Ebenen, in denen jede Fahne von einer nicht-trivialen Elation invariant gelassen wird, Abh. Math. Sem. Univ. Hamburg 29 (1965), 27-76. 8. D. L. Morgan, and T. G. Ostrom, Coordinate systems of some semi-translation planes, Trans. Amer. Math. Soc. 111 (1964), 19-32.

9. T. G. Ostrom, A characterization of the Hughes planes, Canad. J. Math. 17 (1965), 916-922.

10. Collineation groups of semi-translation planes, Pacific J. Math. 15(1965), 273-279.

11. - Derivable nets, Canad. Math. Bull. 8 (1965), 601-613.

12. - The dual Lüneburg planes, Math. Z. 92 (1966), 201-209.

13. - Finite planes with a single ( $p, L)$-transitivity, Arch. Math. 15 (1964).

14. - Replaceable nets, net collineations, and net extensions, Canad. J. Math.

18 (1966), 666-672.

15. - - Semi-translation planes, Trans. Amer. Math. Soc. 111 (1964), 1-18.

16. Some translation planes that are not well known, Washington State University Notes, 1968.

17. — - Transitivities in projective planes, Canad. J. Math. 9 (1957), 389-399.

18. - Vector spaces and construction of finite projective planes, Arch. Math. 19 (1968), 1-25.

19. Proceedings of the projective geometry conference, 1967, University of Illinois, Chicago, 100-120.

Received June 19, 1968, and in revised form February 13, 1970.

UNIVERSITY OF IOWA

IOW A City, IOWA 



\section{PACIFIC JOURNAL OF MATHEMATICS}

\section{EDITORS}

H. SAMELSON

Stanford University

Stanford, California 94305

\section{Richard Pierce}

University of Washington

Seattle, Washington 98105
J. DugundJI

Department of Mathematics

University of Southern California

Los Angeles, California 90007

RICHARD ARENS

University of California

Los Angeles, California 90024

\section{ASSOCIATE EDITORS}

\section{E. F. BeCKenBACH}

B. H. NeUmanN
K. YosHida

\section{SUPPORTING INSTITUTIONS}

\author{
UNIVERSITY OF BRITISH COLUMBIA \\ CALIFORNIA INSTITUTE OF TECHNOLOGY \\ UNIVERSITY OF CALIFORNIA \\ MONTANA STATE UNIVERSITY \\ UNIVERSITY OF NEVADA \\ NEW MEXICO STATE UNIVERSITY \\ OREGON STATE UNIVERSITY \\ UNIVERSITY OF OREGON \\ OSAKA UNIVERSITY \\ UNIVERSITY OF SOUTHERN CALIFORNIA
}

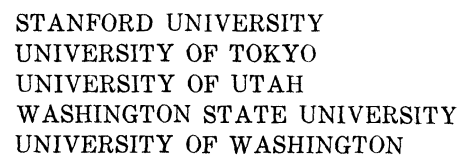

STANFORD UNIVERSITY UNIVERSITY OF TOKYO

UNIVERSITY OF UTAH

WASHINGTON STATE UNIVERSITY

UNIVERSITY OF WASHINGTON

The Supporting Institutions listed above contribute to the cost of publication of this Journal, but they are not owners or publishers and have no responsibility for its content or policies.

Mathematical papers intended for publication in the Pacific Journal of Mathematics should be in typed form or offset-reproduced, (not dittoed), double spaced with large margins. Underline Greek letters in red, German in green, and script in blue. The first paragraph or two must be capable of being used separately as a synopsis of the entire paper. The editorial "we" must not be used in the synopsis, and items of the bibliography should not be cited there unless absolutely necessary, in which case they must be identified by author and Journal, rather than by item number. Manuscripts, in duplicate if possible, may be sent to any one of the four editors. Please classify according to the scheme of Math. Rev. Index to Vol. 39. All other communications to the editors should be addressed to the managing editor, Richard Arens, University of California, Los Angeles, California, 90024.

50 reprints are provided free for each article; additional copies may be obtained at cost in multiples of 50 .

The Pacific Journal of Mathematics is published monthly. Effective with Volume 16 the price per volume (3 numbers) is $\$ 8.00$; single issues, $\$ 3.00$. Special price for current issues to individual faculty members of supporting institutions and to individual members of the American Mathematical Society: $\$ 4.00$ per volume; single issues $\$ 1.50$. Back numbers are available.

Subscriptions, orders for back numbers, and changes of address should be sent to Pacific Journal of Mathematics, 103 Highland Boulevard, Berkeley, California, 94708.

PUBLISHED BY PACIFIC JOURNAL OF MATHEMATICS, A NON-PROFIT CORPORATION

Printed at Kokusai Bunken Insatsusha (International Academic Printing Co., Ltd.), 7-17, Fujimi 2-chome, Chiyoda-ku, Tokyo, Japan. 


\section{Pacific Journal of Mathematics}

\section{Vol. 34, No. 3 \\ July, 1970}

Richard Hindman Bouldin, The peturbation of the singular spectrum

Hugh D. Brunk and Søren Glud Johansen, A generalized Radon-Nikodym derivative .

Henry Werner Davis, F. J. Murray and J. K. Weber, Families of $L_{p}$-spaces

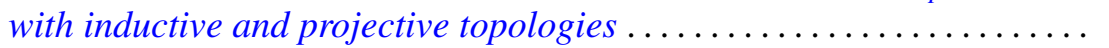

Esmond Ernest Devun, Special semigroups on the two-cell .

Murray Eisenberg and James Howard Hedlund, Expansive automorphisms

of Banach spaces ......................................

Frances F. Gulick, Actions of functions in Banach algebras.

Douglas Harris, Regular-closed spaces and proximities.

Norman Lloyd Johnson, Derivable semi-translation planes . .

Donald E. Knuth, Permutations, matrices, and generalized Young

tableaux..........................................

Herbert Frederick Kreimer, Jr., On the Galois theory of separable

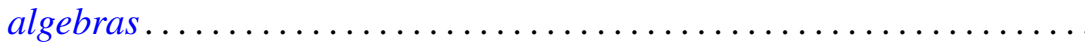

You-Feng Lin and David Alon Rose, Ascoli's theorem for spaces of

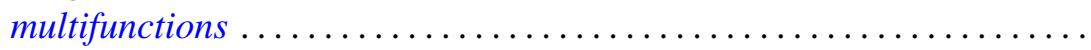

David London, Rearrangement inequalities involving convex functions . . . .

Louis Pigno, A multiplier theorem.

749

Helga Schirmer, Coincidences and fixed points of multifunctions into trees.

755

Richard A. Scoville, Some measure algebras on the integers .

Ralph Edwin Showalter, Local regularity of solutions of Sobolev-Galpern

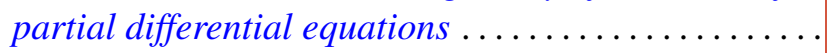

Allan John Sieradski, Twisted self-homotopy equivalences

John H. Smith, On S-units almost generated by S-units of subfields ...

803

Masamichi Takesaki, Algebraic equivalence of locally normal

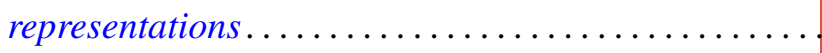

Joseph Earl Valentine, An analogue of Ptolemy's theorem and its converse in

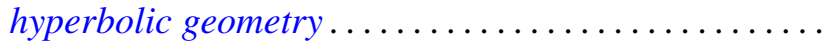

David Lawrence Winter, Solvability of certain p-solvable linear groups of finite order 